\title{
VARIATIONS IN CLINICAL PRESENTATION, NEUROIMAGING AND EEG PATTERNS OF SUBACUTE SCLEROSING PANENCEPHALITIS
}

\author{
Areeba Wasim, Javeria Raza Alvi, Natasha Ghani, Athar Khalily, Zia Ur Rehman, Tipu Sultan \\ Children's Hospital \& Institute of Child Health, Lahore, Pakistan
}

\begin{abstract}
Objective: To determine the variations in clinical presentation, neuroimaging and electroencephalography patterns of subacute sclerosing panencephalitis.

Study Design: Cross-sectional study.

Place and Duration of Study: Children's Hospital \& Institute of Child Health, Lahore, Pakistan, from Jul to Dec 2020.

Methodology: We recruited children presented with clinical features suggestive of subacute sclerosing panencephalitis, along with positive anti-measles antibodies on cerebrospinal fluid. Association between variables was determined to formulate an early diagnosis of subacute sclerosing panencephalitis.

Results: Out of 47 children, 29 were males with a mean age of $6.54 \pm 2.9$ years. Only $23 \%$ were fully immunized against measles, $36.2 \%$ were unvaccinated and $40.4 \%$ received partial immunization. The mean age of measles infection was $1.49 \pm 1.2$ years; the mean interval between measles and onset of SSPE was $4.13 \pm 3$ years. Atypical clinical presentation was seen in $38.3 \%$ with intractable epilepsy $(8.5 \%)$, focal deficit $(8.5 \%)$ and extrapyramidal symptoms $(8.5 \%)$ being commonest followed by coma $(6.4 \%)$, visual loss $(4.3 \%)$ and psychosis $(2.1 \%)$. Neuroimaging was suggestive of cortical hyperintensities in $46.8 \%$ and was normal in $46.8 \%$. Electroencephalography showed burst suppression in $55.3 \%$ and atypical findings in $19.1 \%$. Younger age (1-1.5 years) of measles and unimmunized status were associated with early onset of SSPE with a $p$-value of 0.001 and 0.05 respectively. Non-immunized status was associated with atypical presentation of SSPE ( $p$-value $<0.05)$.

Conclusion: The younger age of measles infection and failure to receive complete immunization led to early onset of Subacute sclerosing panencephalitis with an atypical presentation.
\end{abstract}

Keywords: Anti-measles antibodies, Electroencephalography, Subacute sclerosing panencephalitis.

How to Cite This Article: Wasim A, Alvi JR, Ghani N, Khalily A, Rehman ZU, Sultan T. Variations in Clinical Presentation, Neuroimaging and EEG Patterns of Subacute Sclerosing Panencephalitis. Pak Armed Forces Med J 2021; 71(5): 1839-1843. doi: https://doi.org/10.51253/pafmj.v71i5.6669

This is an Open Access article distributed under the terms of the Creative Commons Attribution License (https://creativecommons.org/licenses/by-nc/4.0/), which permits unrestricted use, distribution, and reproduction in any medium provided the original work is properly cited.

\section{INTRODUCTION}

Subacute sclerosing panencephalitis (SSPE) is a progressive neurodegenerative disorder caused by the persistence of the measles virus in the central nervous system. ${ }^{1}$ The pathogenesis of SSPE is not much elucidated but wild type virus strains are the major culprit. The capacity of wild type viruses to cause SSPE results from certain mutations in their genome, the most common being $M$ protein, resulting in its increased capacity to spread and cause extensive neuronal damage. ${ }^{2}$ It usually occurs 7-10 years after measles infection, but the latent period varies from 1 month to several years. ${ }^{3}$ A shorter latency has been reported in children who contracted measles infection at an earlier $(<2$ years) age. The incidence of SSPE is $18 / 100,000$ in children who had measles younger than 5 years and 1.1/ 100,000 in children with measles after 5 years. ${ }^{4}$

SSPE is a chronic and fatal neurological disorder

Correspondence: Dr Areeba Wasim, Dept of Paediatric Neurology, The Children Hospital \& Institute of Child Health Lahore Pakistan

Received: 29 Apr 2021; revision received: 06 Jul 2021; accepted: 12 Jul 2021 with an unfavourable outcome. The disease has a variable clinical course as fulminant, acute, sub-acute, or chronic. ${ }^{5}$ Clinical presentation varies from typical features of cognitive decline and myoclonic seizures to atypical features like psychosis, focal deficit and visual loss. Having a variable presentation, diagnosis of SSPE need a high index of suspicion with prompt electrophysiological and biochemical investigations. Classically it has a downhill course with high mortality but there may be a transient plateau phase with improvement. ${ }^{6}$ Spontaneous remission and survival beyond 4 years has also been reported. Differential diagnoses include progressive myoclonic epilepsy, viral encephalitis, acquired demyelinating disorders, leukodystrophies, mitochondrial and psychiatric disorders. ${ }^{7}$ Neuroimaging findings are supportive but not specific to SSPE. ${ }^{8}$ A significant association between visual deficits and parieto-occipital signal changes on MRI was found. Electroencephalographic (EEG) findings vary in different stages of SSPE from the disorganized background and burst suppression pattern to the encephalopathic picture. ${ }^{9}$ 
The rationale of the study was to identify variations in clinical presentations, neuroimaging and EEG patterns of patients with SSPE right at presentation. Variable clinical presentations may lead to the diagnostic dilemma and unnecessary investigations especially in developing countries, where measles is endemic and vaccination status is not up to the mark because of poor literacy and socioeconomic status. Given the fact that no such study has been conducted locally, this study will generate evidence for formulating an early diagnosis of SSPE.

\section{METHODOLOGY}

It was a hospital based cross-sectional study conducted in the Paediatric Neurology department at the Children's Hospital and Institute of Child Health, Lahore, from July to December 2020 after obtaining ethics approval from the institutional review board (Certificate number: 2020-115-CHICH). Sample size was taken as 47 using $95 \%$ confidence interval and 5\% margin of error taking incidence of subacute sclerosing panencephalitis as $6 / 100,000$ population using OpenEpi calculator. ${ }^{8}$ Sampling technique was non-probability; purposive type.

Inclusion Criteria: Patients in age group of 1-18 years of either gender with clinical presentation suggestive of SSPE, along with positive anti-measles IgG antibodies on Cerebrospinal fluid ( $>1.5$ IgG liquor assay) were included in study.

Exclusion Criteria: Patients with negative anti-measles antibodies on CSF ( $<1.5$ IgG liquor assay), progressive myoclonic epilepsies, acquired demyelinating disorders, other neurodegenerative disorders, leukodystrophy and mitochondrial disorders were excluded from the study.

SSPE was defined by clinical presentation as typical (Jabbour classification stage I, II, III, IV) or atypical along with positive anti measles anti-bodies on CSF (>1.5 IgG liquor liquor assay). ${ }^{10}$ All patients fulfilling the operational definition of SSPE were enrolled in the study after taking parental consent. Data was recorded on pre-designed proforma including bio-data, demographic features, immunization status as per EPI schedule taking in account number of doses of measles vaccine, age of measles infection in past, age of onset of SSPE and time interval between measles infection and onset of SSPE were documented. Clinical features were recorded in detail as per Jabbour classification stage I: deteriorating school performance, cognitive decline, speech regression, cognitive impairment or memory issues, stage II: myoclonic jerks or dyskinesias, stage
III: non-ambulatory, spasticity or rigidity, stage IV: vegetative state, autonomic failure or decorticate posturing; or the presenting atypical features as isolated psychiatric symptoms, int-ractable epilepsy, dystonia, chorea, focal deficit, ataxia, acute and subacute coma and visual issues. ${ }^{10,11}$ Fundo-scopic findings were recorded as either normal, papilledema, papillitis, necrotising retinitis or optic atrophy. ${ }^{12}$ Neuroimaging findings were recorded as either normal, brain oedema, atrophy or hyperintense signals either in periventricular white matter, gyriform parietooccipital hyperintensities or focal hyperintense signals. ${ }^{8}$ EEG findings were recorded as either in EEG stage A: disorganised background and high voltage delta waves with frontocentral accentuation, stage B: generalised periodic or aperiodic burst of spike and wave followed by suppression, stage C: generalised slowing or the atypical identified features as focal burst suppression, generalised periodic epileptic discharges (GPEDS), status epilepticus or burst of only slow wave discharges followed by suppression. ${ }^{9}$

Data was analysed by using SPSS-20. Numerical variables like age of patients at measles infection, onset of SSPE and time interval between measles and SSPE were taken by mean \pm SD. Qualitative variables like gender, demography, immunization status, clinical and EEG stage along with neuroimaging findings were presented by frequency and percentage. Chi square was used to find association between different variables, $p$-value of $\leq 0.05$ was considered significant.

\section{RESULTS}

A total of 47 children were enrolled in a study out of which $29(61.7 \%)$ were males and $18(38.3 \%)$ were females. The mean age of the patients was $6.54 \pm 2.9$ years. Distribution of age in various subgroups showed that 24 patients $(51.1 \%)$ were between $2-6$ years of age, 19 patients $(40.4 \%)$ between 6-10 years, 3 patients (6.4\%) between $10-14$ years and 1 patient $(2.1 \%)$ between 14-18 years of age. Region-wise, 31 (66\%) belonged to Central Punjab, $13(27.7 \%)$ to Khyber Pakhtunkhwa and $3(6.4 \%)$ to South Punjab. The majority of patients developmentally aged appropriate $46(97.9 \%)$ while only $1(2.1 \%)$ patient was delayed. About vaccination status, $17(36.2 \%)$ were unvaccinated, $19(40.4 \%)$ received only Measles I and II (23.4\%) received both Measles I \& II. The mean age of measles infection was 1.49 \pm 1.2 years; the mean age of onset of SSPE was $6.29 \pm 2.9$ and the mean interval between measles and onset of SSPE was $4.13 \pm 3.0$ year (Table). 
Table: Neuroimaging and Electroencephalography Characteristics of Patients.

\begin{tabular}{l|c}
\hline Characteristics & Values (\%) \\
\hline Neuroimaging Findings, $\mathbf{n}(\%)$ & $22(46.8)$ \\
\hline Normal & $1(2.1)$ \\
Cerebral edema & $2(4.3)$ \\
Cortical atrophy & $22(46.8)$ \\
Non enhancing hyper intense signals & $10(21.3)$ \\
Periventricular hyperintesities & $7(14.9)$ \\
Gyriform parieto-temporal hyperintensities & $2(4.3)$ \\
Occipital hyperintensities & $2(4.3)$ \\
Focal cortical hyperintensities & $1(2.1)$ \\
White matter \& brainstem signals & \\
\hline EEG Findings, $\mathbf{n}$ (\%) & \\
\hline Stage A: & $10(21.3)$ \\
High voltage delta waves with fronto-central & $26(55.3)$ \\
accentuation & $15(31.9)$ \\
Stage B: & $11(23.4)$ \\
Generalized Burst suppression pattern & $2(4.3)$ \\
Periodic & $9(19.1)$ \\
Aperiodic & $1(2.1)$ \\
Stage C: & $2(4.3)$ \\
Generalized slow waves & $1(2.1)$ \\
Atypical EEG findings: & $4(8.5)$ \\
Status epilepticus & $1(2.1)$ \\
Focal Burst suppression & Epileptic \\
Burst of slow waves followed by suppression & \\
GPEDs & \\
Multifocal discharges & \\
\hline SD:Standard Deviation; GPED: Generalized Periodic \\
Discharges. & \\
\hline
\end{tabular}

Regarding clinical presentation, the majority of the patients were of Stage II $(36.2 \%)$ and atypical presentation $(38.3 \%)$. Out of 47 children, $6(12.8 \%)$ were of Stage I with cognitive decline in 1 patient $(2.1 \%)$, memory impairment in 2 patients $(4.3 \%)$ and $3(6.4 \%)$ patients were having combined features of speech and behavioural issues. Stage II was seen in 17 (36.2\%) patients with 15 (31.9\%) having myoclonic jerks. Only $1(2.1 \%)$ had dyskinesia and $1(2.1 \%)$ had both jerks and dyskinesia. Stage III was seen in $5(10.6 \%)$ with loss of ambulation in $3(6.4 \%)$ and spasticity in $2(4.3 \%)$ patients. Stage IV was seen in $1(2.1 \%)$ manifested by vegetative state. Atypical clinical presentation was seen in $18(38.3 \%)$ patients; the breakout is shown in Figure-1. Fundoscopic findings were normal in 26 $(55.3 \%)$ patients. Rest showed papilledema in 10 $(21.3 \%)$, optic atrophy in $8(17 \%)$, papillitis in $2(4.3 \%)$ and necrotizing retinitis in $1(2.1 \%)$ patients.

Among enrolled patients, the vaccination status with the majority being partially vaccinated $(40 \%)$ and unvaccinated $(36 \%)$ had an early clinical presentation with SSPE with a significant $p$-value $<0.05$. The patients who had measles infection between 1-1.5 years had a shorter interval between measles and onset of
SSPE i.e. $4.13 \pm 3$ years with a significant $p$-value of $<0.001$. There was a significant association between the age of patients and fundus findings with a $p$-value $<0.001$. Neuroimaging showed non-enhancing hyperintense signals in $46.8 \%$ cases out of which $21.3 \%$ had periventricular hyperintensities and $4.3 \%$ had focal abnormal signals as shown in Figure-2A \& B. Occipital hyperintensities $(4.3 \%)$ were seen in patients with isolated visual loss. The majority of the patients had EEG stage B $(55.3 \%)$ at presentation with periodic burst suppression pattern $(31.9 \%)$ even with focal physical and neuroimaging findings as shown in Figure-2, in a patient with the left focal deficit, MRI showed T2W right-sided white matter hyper-intense signal along
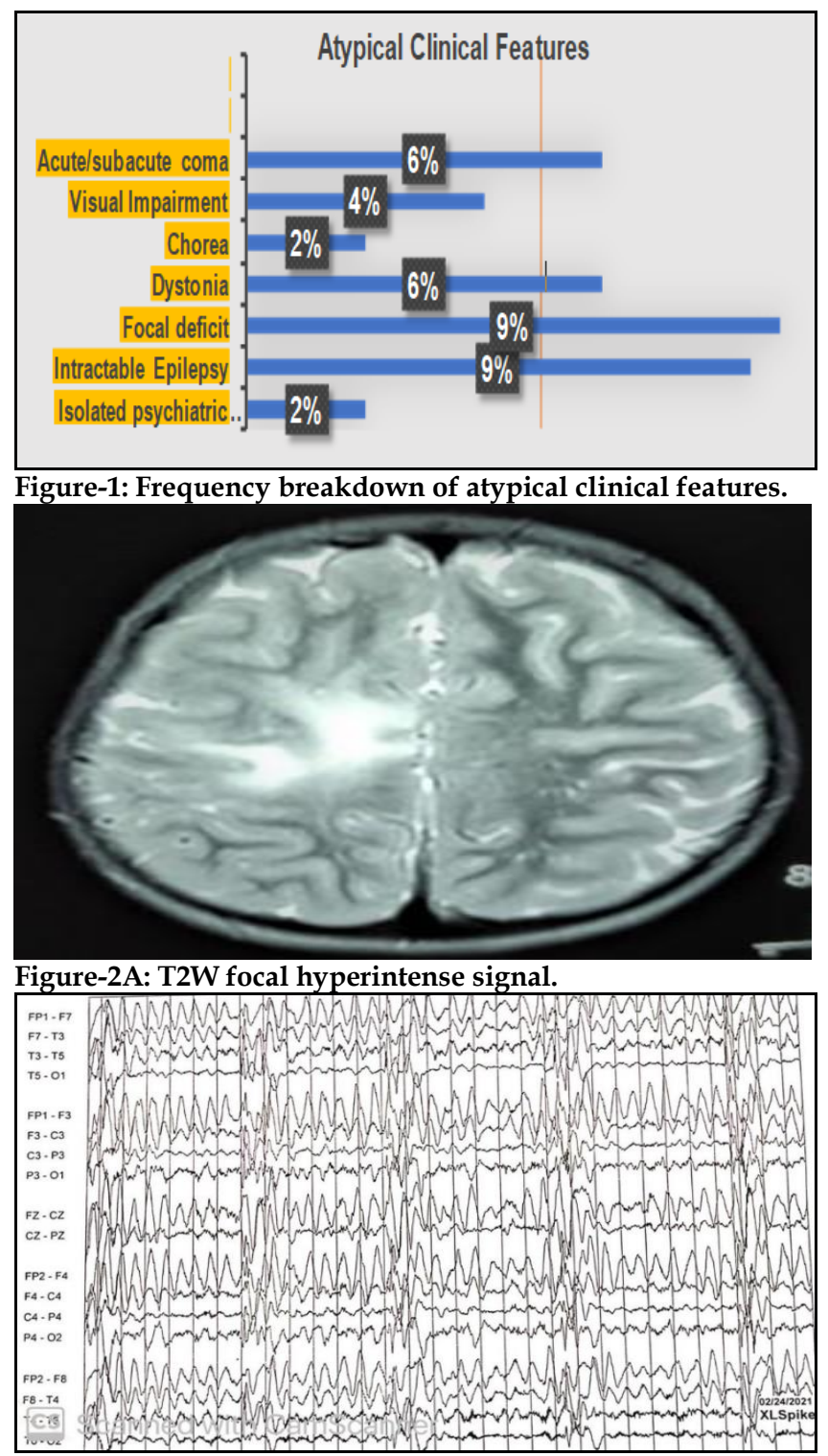

Figure-2B: burst suppression pattern. 
with generalized burst suppression pattern-reason for suspecting SSPE in this case EEG stage B was seen in $55.3 \%$ cases amongst which with periodic burst suppression was present in $31.9 \%$ cases. Atypical EEG features were seen in $19.1 \%$ of cases; $8.5 \%$ showed GPEDs and 2.1\% had status epilepticus. A relationship was seen between the clinical stage and EEG stage but we were unable to establish different clinical findings with each EEG stage.

\section{DISCUSSION}

Subacute sclerosing panencephalitis is a devastating neurodegenerative disease with a downhill course and marked difference in the incidence of disease as per demographic profile. In Pakistan, there is no data registry to date for neither measles nor SSPE, the reason being lack of confirmatory tests for measles infection and specialised testing like EEG and antimeasles antibodies at many healthcare centres ${ }^{13}$. In our study, unvaccinated and partially vaccinated patients had the early age of onset of SSPE with quite variable presentations. Osama et al, reported that during 2000-2017, Measles vaccine I and II coverage in Pakistan increased substantially, to $76 \%$ and $45 \%$, respectively, but remained well below the WHO-recommended level of $\geq 95 \%$. In addition, large-scale measles outbreaks occurred during 2012-2014 and 2016-2018, revealing coverage gaps from the routine immunization services. ${ }^{14}$ Aulakh et al, reported SSPE in a toddler of 2.5 years with a history of measles just 4 months back, favouring results of our study in the aspect that patients with younger age of measles had short incubation period i.e. the youngest being 2 years in our study. ${ }^{15}$ Previously SSPE was considered to have a longer incubation period of at least 8-10 years after measles infection as evident from earlier studies done in Pakistan and India while two of our SSPE patients had no recall of measles infection. ${ }^{16,17}$

Rafique et al reported demographic and clinical profile of SSPE in their retrospective study with the majority of the patients presented with seizures i.e. myoclonic seizures $(74.4 \%)$, generalised tonic-clonic (21.\%) and generalised tonic $(4.6 \%)$ on contrary to our study where $31.9 \%$ had myoclonic seizures right at presentation. Atypical clinical features were documented in $38.3 \%$ of our patients constituting the major bulk of clinical manifestation along with Jabbour clinical stage II. Gokoglu A and Gozdas HT reported adultonset SSPE with Neuropsychiatric Symptoms along with the negative history of measles exposure, which was confirmed by typical EEG pattern and positive antimeasles antibodies in CSF. ${ }^{18}$ Isolated Psychiatric manifestations are very rare in the pediatric population with no prior study documented them as sole presentation while our one patient had sole neuro-psychiatric symptoms of low mood, disinterest in play and school after the demise of the family member along with focal temporal lesion and characteristic EEG, later confirmed by antimeasles anti-bodies. Sharma et al reported subacute sclerosing panencephalitis (SSPE) during the postpartum period with cortical vision loss and parietooccipital white matter T2/ FLAIR hyperintensities simulating posterior reversible encephalopathy. ${ }^{19}$ We had two patients at our centre with central visual loss and occipital hyperintensities with no recall for measles infection. Tripathy et al, reported ophthalmological findings as a means to dia-gnose SSPE with aid of ocular coherence tomography and ultra field imaging. ${ }^{12}$ They found optic disc pallor and irregular pigmentary macular scar later progressed to retinitis and intraretinal haemorrhage associated with an increase in the frequency of myoclonic seizures while $17 \%$ of SSPE patients with us had optic atrophy and $2.1 \%$ had necrotising retinitis.

Jafri et al studied the MRI spectrum of SSPE patients with the majority having diffused white matter abnormalities and normal MRI in the initial stages of SSPE. ${ }^{8}$ In our study, $46.8 \%$ of patients had hyperintense signals out of which $21 \%$ had periventricular signals and $18 \%$ had cortical abnormalities. Das et al reported adolescents of SSPE having remarkable asymmetric pialgyral enhancements, which were misinterpreted as tubercular infection. ${ }^{20}$ Hasirci reported late development of occipital hyperintensities after 3 months of the onset of visual symptoms, along with multilocular narrowing of medium and small vessels on diffusion subtraction angiography while our two patients with visual loss had occipital hyperintense signals right at presentation. ${ }^{21}$ Vilas et al, reported diffuse demyelinating leukoencephalopathy among atypical presentations of SSPE while we had $4.3 \%$ patients of each focal white matter and occipital hyperintensities. $^{22}$ MR spectroscopy of this focal cortical lesion was suggestive of choline peak.

Rabanese et al, reported periodic burst suppression $(58 \%)$, generalised spike $(15 \%)$ and slow-wave discharges $(30 \%)$ in SSPE patients while describing their demography along with clinical and imaging findings. ${ }^{23}$ In our study, $55.3 \%$ of patients had had EEG stage B; breakdown of which showed periodicity in $31.9 \%$ and aperiodic discharges in $23.4 \%$. Singhi et al 
reported disorganized background with generalized spike-wave discharges 2-2.5/second occupying more than $80 \%$ of tracing, consistent with non-convulsive status epilepticus (NCSE) while our one patient with cognitive decline and intermittent lapses in consciousness had NCSE. ${ }^{24}$

In our study, we presented a comprehensive report of both the typical and atypical features in clinical spectrum, neuroimaging and EEG patterns of SSPE patients, right at presentation in a specialised paediatric neurology centre in Pakistan.

\section{LIMITATION OF STUDY}

This study did not identify the nature of the progression of disease and response to antiviral medication, as we did not record the outcome of patients.

\section{CONCLUSION}

The younger age of measles infection and failure to receive complete immunization led to the early onset of SSPE with an atypical presentation. With this changing epidemiological trend, a high index of suspicion is required to identify SSPE with atypical presentation including very early onset, shorter latency between postnatally acquired measles and SSPE onset along with atypical neuroimaging and EEG spectrum. The younger age of measles infection poses earlier administration of the vaccine in endemic areas.

\section{Conflict of Interest: None.}

\section{Authors' Contribution}

AW: Concept, design, data collection, data analysis, manuscript writing, JRA: Data collection, data analysis, NG: data collection, AK: data collection, ZUR: manuscript review and editing, TS: manuscript review, editing, and final approval

\section{REFERENCES}

1. Jafri S, Kumar R. Subacute sclerosing panencephalitis-current perspectives. Pediatr Health Med Ther 2018; 2018(9): 67-71.

2. Reiss CS. In: Neurotropic Viral Infections. $2^{\text {nd }}$ ed. Reiss CS, editor. Switzerland: Springer International Publishing; 2016, [Internet] Available from: https://www.springer.com/gp/ book/9783319331881

3. Cruzado D, Masserey-Spicher V, Roux L, Delavelle J, Picard F, Haenggeli CA. Early-onset and rapidly progressive subacute sclerosing panencephalitis after congenital measles infection. Eur J Pediatr 2002; 161(8): 438-441.

4. Gutierrez J, Issacson RS. Subacute sclerosing panencephalitis: an update. Dev Med Child Neurol 2010; 52(10): 901-907.

5. Malik MA, Saeed M, Qureshi AU, Ahmed M, Akram M. Predictors of the clinical course of subacute sclerosing panencephalitis. J Coll Physicians Surg Pak 2010; 20(10): 671-674.

6. Öztürk A, Gürses C, Baykan B, Gökyiğit A. Subacute sclerosing panencephalitis: clinical and magnetic resonance imaging evaluation of 36 patients. J Child Neurol 2002; 17(1): 25-29.
7. Ichiyama T. Clinical course and findings in and differential diagnosis of subacute sclerosing panencephalitis. Nihon Rinsho 2007; 65(8): 1481-1482.

8. Jafri SK, Husen Y, Ahmed K, Ibrahim SH. The spectrum of MRI brain findings in subacute sclerosing panencephalitis. Asia Pac J Clin Trials Nerv Syst Dis 2017; 2(4): 124-30.

9. Velmurugan J, Sinha S, Nagappa M, Mariyappa N, Bindu PS, Ravi JS, et al. Combined MEG-EEG source localisation in patients with subacute sclerosing panencephalitis. Neurol Sci 2016; 37(8): 1221-1231.

10. Jabbour J, Duenas D, Modlin J. SSPE-clinical staging, course, and frequency. Arch Neurol 1975; 32(7): 493-494.

11. Jović NJ. Epilepsy in children with subacute sclerosing panencephalitis. Srp Arh Celok Lek 2013; 141(7-8): 434-440.

12. Tripathy K, Chawla R, Mittal K, Farmania R, Venkatesh P, Gulati S. Ophthalmic examination as a means to diagnose subacute sclerosing panencephalitis: an optical coherence tomography and ultrawide field imaging evaluation. Eye Vis 2017; 4(1): 1-5.

13. Ibrahim SH, Amjad N, Saleem AF, Chand P, Rafique A, Humayun KN. The Upsurge of SSPE-a reflection of national measles immunization status in Pakistan. J Trop Pediatr. 2014; 60(6): 449-453.

14. Osama M, Goodson L, Chandio AK, Rana MS, Hasan Q, Teleb N, et al. Progress towards measles elimination-Pakistan, 2000-2018, [Internet] Available from: https://www.cdc.gov/mmwr/ volumes/68/wr/mm6822a4.htm. [Accessed on June 30, 2021]

15. Aulakh R, Tiwari A. Subacute sclerosing panencephalitis in a toddler: changing epidemiological trends. Case Rep Pediatr 2013; 2013(2): 341462.

16. Jagtap SA, Nair MD, Kambale HJ. Subacute sclerosing panencephalitis: A clinical appraisal. Ann Indian Acad Neurol 2013 (10); 16(4): 231-233.

17. Rafique A, Amjad N, Chand P, Zaidi SS, Rana MS, Ahmed K, et al. Subacute sclerosing panencephalitis: clinical and demographic characteristics J Coll Physicians Surg Pak. 2014; 24(8): 557560.

18. Gokoglu A, Gozdas HT. Adult-onset subacute sclerosing panencephalitis presented with neuropsychiatric symptoms. J Coll Physicians Surg Pak 2019; 29(Suppl-1): S29-S30

19. Sharma PK, Garg RK, Singla S. Case report: subacute sclerosing panencephalitis in pregnancy mimicking posterior reversible encephalopathy syndrome. Am J Trop Med Hyg 2020; 102(3): 634-636.

20. Das B, Goyal MK, Mohdi M, Mehta S, Chakravarthi S, Lal V, et al. Atypical magnetic resonance imaging features in subacute sclerosing panencephalitis. Ann Indian Acad Neurol. 2016(4-6); 19(2): 275-276

21. Hasirci BR, Agircan D, Okay M, Orhan A, Kocer A. Subacute sclerosing panencephalitis with atypical clinical and MRI findings. Türk Beyin Damar Hastalıkları Dergisi 2016; 22(1): 25-28.

22. Vilas D, Becerra JL, Lozano M, Soriano A, Matas L, Martro E, et al. Atypical presentation of adult-age onset subacute sclerosing panencephalitis. Rev Neurol 2012; 54(1): 60-61.

23. Rabanese AC, Cruz-Urbi CM. Demographic, clinical, imaging and EEG characteristics of subacute sclerosing panencephalitis. Eur J Pediatr Neurol 2017; 21(1): 110-116.

24. Singhi P, Saini AG, Sahu JK. Non-Convulsive status epilepticus on electroencephalography: an atypical presentation of subacute sclerosing panencephalitis. Case Report Pediar 2012; 10(1): 1155. 